\title{
Multiplexed detection of pathogen DNA with DNA-based fluorescence nanobarcodes
}

\author{
Yougen Li, Yen Thi Hong Cu \& Dan Luo
}

Rapid, multiplexed, sensitive and specific molecular detection is of great demand in gene profiling, drug screening, clinical diagnostics and environmental analysis ${ }^{1-3}$. One of the major challenges in multiplexed analysis is to identify each specific reaction with a distinct label or 'code' 4 . Two encoding strategies are currently used: positional encoding, in which every potential reaction is preassigned a particular position on a solid-phase support such as a DNA microarray ${ }^{5-8}$, and reaction encoding, where every possible reaction is uniquely tagged with a code that is most often optical or particle based $^{4,9-13}$. The micrometer size, polydispersity, complex fabrication process and nonbiocompatibility of current codes limit their usability $1,4,12$. Here we demonstrate the synthesis of dendrimer-like DNA-based, fluorescence-intensity-coded nanobarcodes, which contain a built-in code and a probe for molecular recognition. Their application to multiplexed detection of the DNA of several pathogens is first shown using fluorescence microscopy and dot blotting, and further demonstrated using flow cytometry that resulted in detection that was sensitive (attomole) and rapid.

Recently, dendrimer-like DNA (DL-DNA) nanostructures have been synthesized by our group ${ }^{14}$. The multivalent and anisotropic properties of DL-DNA were used here as fluorescent dye carriers (that is, scaffoldings) to construct fluorescence-intensity-encoded nanobarcodes. We first synthesized fluorescence-labeled Y-shaped DNA (Y-DNA), where each Y-DNA consisted of three oligonucleotide components that were complementary to each other. One of the oligonucleotides had a sticky end, and the other two were labeled with either fluorophore(s) or a molecular probe. After hybridization, these oligonucleotides formed a fluorescence-labeled Y-DNA (Fig. 1a) that was used as a peripheral outermost layer of DL-DNA to construct fluorescence-labeled DNA nanostructures. Since both dye type and dye number can be precisely controlled, multicolor fluorescenceintensity-encoded nanobarcodes could be fabricated (Fig. 1b). The decoding is based on the different ratios of different fluorescent dyes, independent of the dye positions (Fig. 1c). During the construction of DNA nanobarcodes, molecular probes were linked to the free reactive ends of DL-DNA. A myriad of DNA-manipulation enzyme tools ${ }^{15}$ makes it very easy to attach molecular probes (e.g., DNA or RNA probes, or even antibodies) to DNA nanobarcodes. Consequently, the resultant DNA nanobarcodes not only had coding capacity, but also contained molecular recognition elements that could be used for molecular detection.

Two types of fluorescent dyes, Alexa Fluor 488 and BODIPY 630/ 650, were used to label DNA (see Supplementary Tables 1 and 2 online). The fluorescence-labeled Y-DNA (see Supplementary Table 3 online) was ligated to other Y-DNAs via complementary sticky ends. Five nanobarcodes, 4G1R, 2G1R, 1G1R, 1G2R and 1G4R were constructed, where the number refers to the quantity of each dye molecule on one nanobarcode (see Supplementary Table 4 online and Fig. 1c).

The resultant nanobarcodes were evaluated using agarose gel electrophoresis. Oligonucleotides labeled with either Alexa alone or BODIPY alone were controls (Fig. 1d, lanes 1 and 7, respectively); the obvious color changes from green to yellow to red (Fig. 1d, lanes 2 to 6) indicated the formation of the expected different nanobarcodes, a result further confirmed by the electrophoretic mobility shift of DNA nanobarcodes relative to the starting oligonucleotides (Fig. 1d and Supplementary Fig. 1a online). The formation of dendritic DNA nanobarcodes was also confirmed by the generation of oligonucleotides whose lengths differed from the starting oligonucleotides, which were revealed by denaturing agarose gel electrophoresis (see Supplementary Figs. $\mathbf{1 b}$ and 2 online). Details of the evaluation of DNA nanostructures using gel electrophoresis can be found in a previous publication ${ }^{14}$.

The diameter of DNA nanobarcodes was $<30 \mathrm{~nm}$, which is far below the detection limit of optical microscopy. Polystyrene microbeads $(5.5 \mu \mathrm{m}$ diameter) were thus used to amplify the fluorescence signals for imaging and molecular detection. The microbead-based amplification strategy and detection format are shown in Figure 2a. In this arrangement, two sets of single-stranded (ss)DNA probes were used. The first set (capture probes) was biotin labeled and immobilized onto avidin-functionalized microbeads. Note that each batch of microbeads was attached with only one type of capture probe, which was complementary to a part of a particular target DNA (that is, sample DNA to be detected). Multiple types of microbeads were then pooled together to form a library of microbeads. The second set of ssDNA probes (report probes) was coupled to specific nanobarcodes, where each report probe was complementary to another part of the particular target DNA and thus was able to be hybridized onto a specific microbead in the presence of the target DNA via a sandwiched hybridization (Fig. 2a). Because each microbead could accommodate a large number of sandwiched complexes, fluorescence signals from nanobarcodes 
were amplified. In the assay, the sample containing multiple types of unknown DNA (that is, target DNA) was mixed with microbeads in suspension, and captured onto the microbeads containing their corresponding capture probe sequences presented on the bead surface through DNA hybridization. A solution containing a nanobarcode library was then added. Since each nanobarcode was connected with a particular report probe, which in turn hybridized to another portion of the target DNA, nanobarcodes were specifically bound to corresponding microbeads. The resultant microbeads were first evaluated indivi-
dually by fluorescence microscopy, and the overlay color images are shown in Figure 2b, following a similar color-processing approach reported previously ${ }^{16}$. Even with the naked eye, five different nanobarcodes could be distinguished from Figure $\mathbf{2 b}$. The sample mixture, which consisted of DNA from four targets (Bacillus anthracis ${ }^{17}$, Francisella tularensis ${ }^{18}$, Ebola virus ${ }^{19}$ and SARS coronavirus ${ }^{20}$ ), was analyzed with nanobarcodes, and four different pseudocolors were revealed in the overlay image (Fig. 2c). Compared to Figure $2 \mathbf{b}$, we found that four different nanobarcodes, 4G1R, 2G1R, 1G1R and 1G4R, were bound to different microbeads as designed. With the preassigned barcode library (see Supplementary Table 5 online), we concluded

a

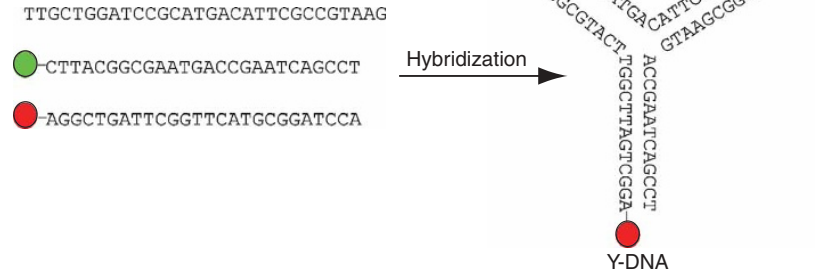

b that the samples contained characteristic DNA from four pathogens: B. anthracis, F. tularensis, Ebola and SARS. The quantitative decoding results at the population level are also shown (see Supplementary Fig. 3 online). Taken together, with one nanobarcode (e.g., 1G1R) serving as a reference, other nanobarcodes could be easily decoded.

Nanobarcodes can also be used for blotting-based detection (Southern, northern and western). Here we used dot blotting to demonstrate this potential. Six samples, including the controls of 27-mer ssDNA with irrelevant sequences and a 6.1-kb plasmid DNA, were first blotted onto a membrane; all five types of nanobarcodes (see Supplementary Table 4 online) were then used to hybridize onto target DNA for detection (Fig. 3a). Simultaneous detection of four colors on the resultant membrane (Fig. 3b) indicated four DNA targets, which were subsequently identified by referring to a preassigned decoding library. As expected, no fluorescent signals were detected in the two control spots, suggesting nanobarcode-based detection is highly specific. In addition, a barcode was similarly used to successfully detect a pathogen genomic DNA (Mycobacterium avium subsp. paratuberculosis) extracted from animal feces in the presence of lysates or serum (data not shown).

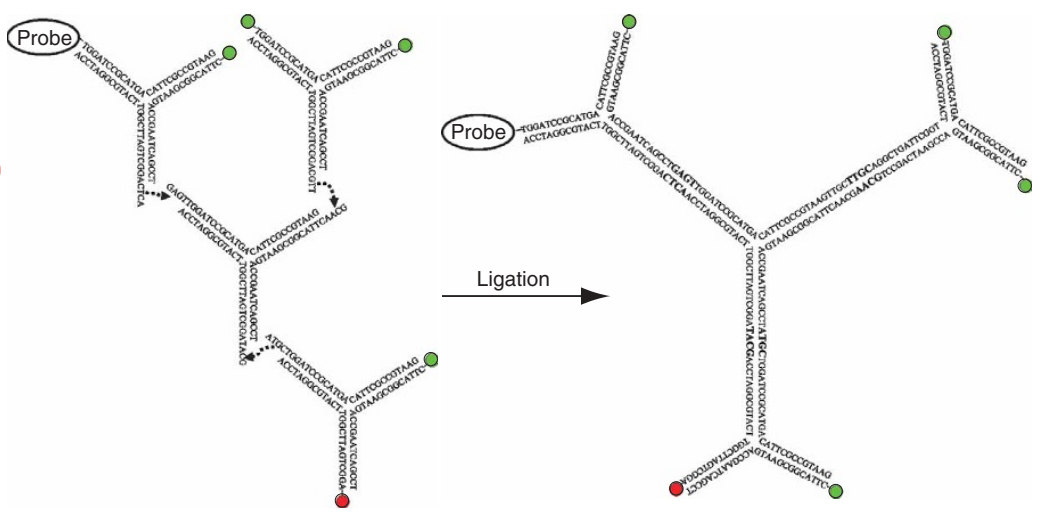

Figure 1 Synthesis of nanobarcodes. (a) Schematic illustration of synthesis of a typical Y-DNA-based nanobarcode building block. Three starting oligonucleotide components were partially complementary to each other as indicated in the drawing. One oligonucleotide possessed a sticky end, another one was labeled with a fluorescent dye and the third one was labeled with a fluorescent dye or a probe depending on the experimental design. (b) Schematic illustration of the construction of a typical DL-DNA-based nanobarcode. The nanobarcode building blocks were covalently linked with each other through complementary sticky-end ligations. (c) Schematic illustration of barcode decoding. The nanobarcodes 4G1R, 2G1R, 1G1R, 1G2R and 1G4R were decoded based on the ratio of fluorescence intensity. A molecular recognition element, a probe, was also attached to each nanobarcode. The resultant nanobarcodes possess not only coding capability and capacity, but also molecular sensing ability. With a preassigned code library (see Supplementary Table $\mathbf{5}$ online), the nanobarcodes could be used for molecular detection. (d) The real color of nanobarcodes in an agarose gel illuminated with a strong UV light. Lanes 1 and 7 are Alexa Fluor 488-labeled starting oligonucleotide component and Bodipy 630/650-labeled starting oligonucleotide component, respectively. Lanes 2, 3, 4, 5, 6 are nano-barcodes 4G1R, 2G1R, 1G1R, 1G2R and 1G4R, respectively. 
a

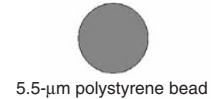

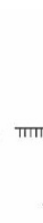
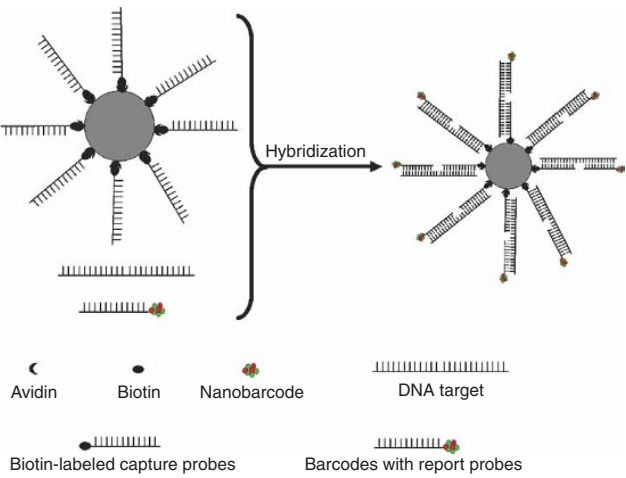

Ешшшшшшшшше DNA target Barcodes with report probes

Figure 2 Microbead-based DNA detection using fluorescence microscopy. (a) Schematic drawing of a sandwiched DNA nanobarcode whose signal is amplified from polystyrene microbeads. Briefly, biotin-labeled capture probes were attached to avidin-functionalized polystyrene microbeads. Each batch of microbeads had only one type of capture probe before all batches were pooled together. DNA targets (that is, control or unknown samples) were then captured by specific microbeads first. Each report probe, which was linked to a particular nanobarcode, was designed to be complementary to another part of a specific target DNA and thus was able to be hybridized onto a specific microbead. Since each microbead bound a large amount of sandwiched

complexes (that is, capture probes/target DNA/report probes/nanobarcodes), of nanobarcodes from individual microbeads. (c) Multiple target detection (a total of four targets) was achieved via a two-colored fluorescence microscope using DNA nanobarcodes and microbeads. All scale bars, $5 \mu \mathrm{m}$.

Flow cytometry is a powerful tool for multiplexed molecular detection $^{21,22}$. The application of nanobarcodes to multiplexed molecular detection was also demonstrated using flow cytometry. The fluorescence intensity ratio is the basis for the decoding of any nanobarcodes $a \mathrm{G} b \mathrm{R}$ (see Supplementary Note $\mathbf{1}$ online for the mathematical processing of flow data). To simultaneously detect DNA from multiple pathogens, we made a solution containing mixtures of DNA from three pathogens with one irrelevant DNA sample as a negative control. This solution was treated as a sample with unknown pathogen DNA. Samples were allowed to bind to the microbeads without saturation. All five types of nanobarcodes (see Supplementary Table 4 online) were then added into a bead suspension. After the hybridization was complete, the fluorescence intensity ratio was measured for each microbead by flow cytometry (Fig. 4b). From the intercepts on the flow plots, the $K$ values (the total fluorescence intensity ratio between red and green fluorophores, see Supplementary Note 1 online) of each line (top to bottom) were determined to be 11, 44 and 180, and their code numbers were thus calculated to be around 4.0, 1.0 and 0.25 , respectively. Therefore, the detected nanobarcodes were 4G1R, 1G1R and 1G4R. The same detection experiments were repeated four times and the statistical data showed that the coding nanobarcodes were indeed 4G1R, 1G1R and 1G4R (see Supplementary Fig. 4 online). After referring to the preassigned barcode library (see Supplementary Table 5 online), we concluded that the unknown samples contained DNA from three pathogenic species: $B$. anthracis, Ebola and SARS. The detection limit was $6.2 \times 10^{-16}$ mole or 620 attomole. The final detection step was completed within $30 \mathrm{~s}$. The success of simultaneously identifying multiple pathogen DNA with attomole sensitivity within a very short period of time ( $<1 \mathrm{~min}$ ) demonstrated the potential of nanobarcodes for multiplexed molecular detection. Theoretical and practical coding capacity is discussed in Supplementary Note 2 online. b
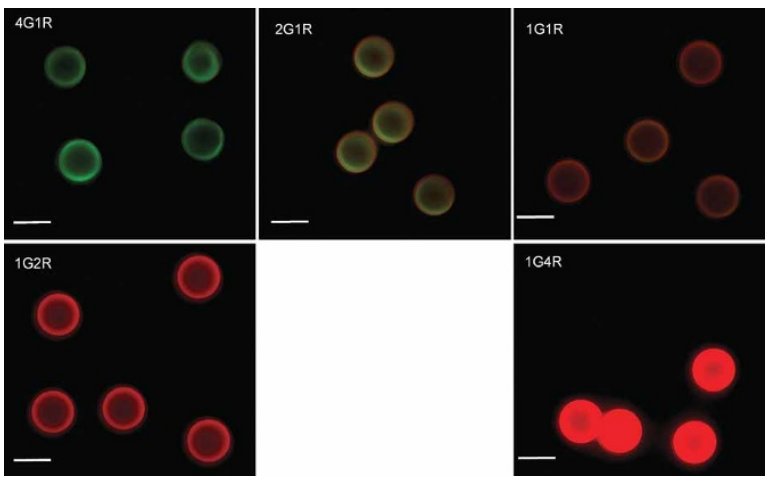

C

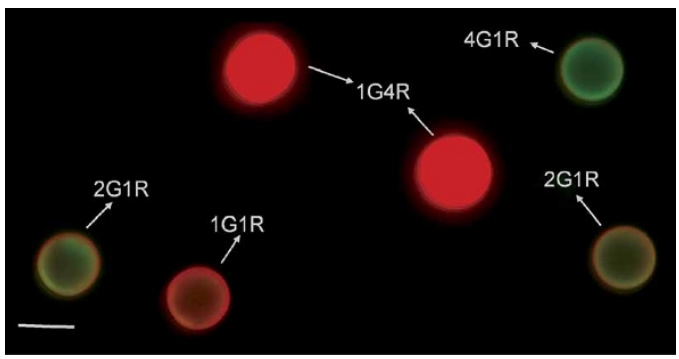

The successful synthesis and application of DL-DNA based nanobarcodes reveals two novel concepts: (i) multiplexed detection can be achieved by detecting different fluorescence intensity ratios instead of different fluorescent colors, and (ii) DL-DNA can be used as both structural scaffolding and functional probes. Although DNA-based signal amplifications have been reported previously (e.g., with

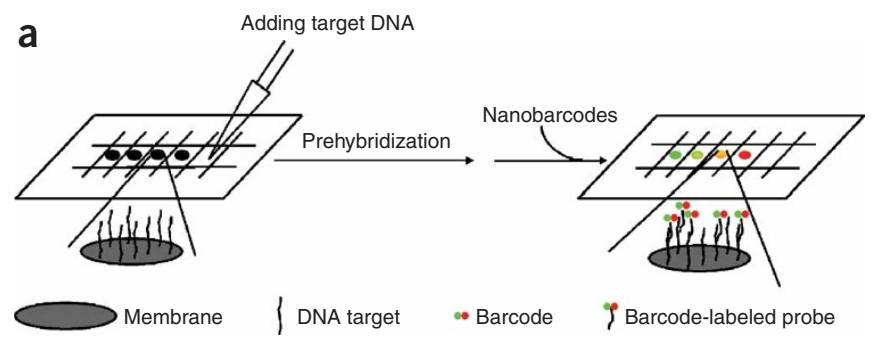

b

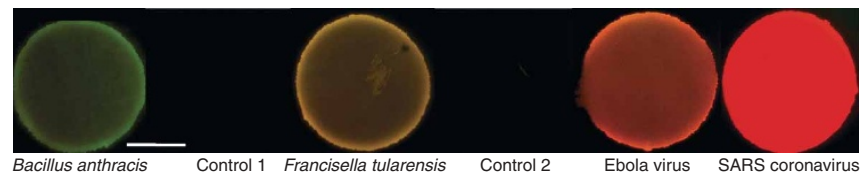

Figure 3 DNA blotting assay with nanobarcodes. (a) Schematic drawing of a dot-blotting detection of multiple DNA targets with nanobarcodes. Target DNA molecules were manually blotted onto a nylon-membrane. After prehybridization and blocking, a library of nanobarcode mixture was loaded onto the membrane. Through specific hybridizations with report probes that were functionalized with nanobarcodes, target DNA molecules were detected using a fluorescence reader, scanner or microscope. (b) DNA from multiple pathogens (four in total) were detected simultaneously using nanobarcodes. Control 1 was a 27-mer ssDNA with unrelated sequences and control 2 was a plasmid DNA, pVAX1/lacZ. Scale bar, $1 \mathrm{~mm}$. 
chemical cross-linked dendritic $\mathrm{DNA}^{23-27}$ or backbone-branched oligonucleotides ${ }^{28,29}$ ), to our knowledge no one else has demonstrated successful multiplexed detection using DNA nanobarcodes, whose fluorescence intensity ratios were precisely controlled at an individual molecular level; at present such tight control of color-conjugations can be achieved only with anisotropic, multivalent carriers, such as DL-DNA. In addition, the DNA scaffold makes our nanobarcodes biocompatible and thus has the potential to be applied in vivo after solving degradation problems. Another advantage of using DL-DNA as a scaffolding material lies in the existence of DNA modifying enzymes. Functional elements can be easily introduced either before (e.g., the molecular recognition elements can be linked to the oligonucleotide components) or after nanobarcode synthesis. Moreover, the nano-scale size of our nanobarcodes ensures easier target access in vivo or in situ when compared to current microsized codes ${ }^{4,9-12}$. Note that the in situ possibility can be realized only when a spatial resolution can be achieved to distinguish individual 을 barcodes (for example, by DL-DNA that is larger than $150 \mathrm{~nm}$ ).

The use of common and commercially available fluorophores does not require special equipment for detection, effectively expanding the power of traditional microscopy. In addition, this technique could also substitute both isotope and fluorochrome labeling for blotting-based, multiplexed detection without resorting to multiple runs or repeated probe stripping, as practiced at present. Furthermore, nanobarcodes make fast, sensitive and multiplexed detection possible in a flow cytometer that can detect only two colors.

In summary, we have developed a DL-DNA-based nanobarcode technology, whose applications have been demonstrated in fluorescence microscopy, dot blotting and flow cytometry. The technology has the following advantages: (i) DNA, in particular the DL-DNA, can be used as both a structural scaffolding and a functional probe; (ii) multiplexed molecular sensing relies on the detection of precise fluorescent color ratios instead of the detection of single colors; and (iii) the DNA-based, multiplexed sensing platform nanotechnology can be applied to almost any fluorescence-based detection system.

\section{METHODS}

Synthesis of DNA nanobarcodes. Each nanobarcode building block, the fluorescence-labeled Y-DNA, consisted of three oligonucleotides (see Supplementary Tables 1 and 2 online), one of which had a nonpalindromic sticky end whereas the other two were either fluorescence labeled (with Alexa Fluor $488\left(\mathrm{E}_{\mathrm{x}}=495 \mathrm{~nm}\right.$ and $\left.\mathrm{E}_{\mathrm{m}}=519 \mathrm{~nm}\right)$ or BODIPY $630 / 650\left(\mathrm{E}_{\mathrm{x}}=625 \mathrm{~nm}\right.$ and $\left.\mathrm{E}_{\mathrm{m}}=640 \mathrm{~nm}\right)$ ) or attached to a DNA probe. The three DNA components were partially complementary to each other. After hybridization, they formed Y-DNA, which was used as the outermost peripheral layer of the nanobarcodes. Other nonfluorescence labeled Y-DNA was used to link fluorescence-labeled Y-DNA together. All Y-DNA were ligated to each other via their complementary sticky ends to form fluorescence-labeled dendritic nanostructures (nanobarcodes) (Fig. 1b). The details of DNA sequence design, synthesis of Y-DNA and fabrication of dendrimer-like DNA have been published ${ }^{14}$.

Gel electrophoresis. The DNA nanobarcodes were run in a 3\% agarose ready gel (Bio-Rad) at $85 \mathrm{~V}$ at $25^{\circ} \mathrm{C}$ in Tris-acetate-EDTA (TAE) buffer $(40 \mathrm{mM}$ Tris, $20 \mathrm{mM}$ acetic acid and $1 \mathrm{mM}$ EDTA, pH 8.0, Bio-Rad). After a true color picture of the gel was taken using a digital camera under strong UV illumination, it was stained with $0.5 \mu \mathrm{g} / \mathrm{ml}$ of ethidium bromide in TAE buffer. DNA denaturation was performed ${ }^{14}$. Briefly, a 10 pmol DNA sample in a denaturing buffer $(10 \mathrm{mM}$ EDTA, $25 \mathrm{mM} \mathrm{NaOH})$ was heated at $95^{\circ} \mathrm{C}$ for $2 \mathrm{~min}$ and then immediately cooled down in a $-20^{\circ} \mathrm{C}$ freezer. The denatured DNA sample was run through a $3 \%$ agarose gel at $50 \mathrm{~V}$ for $10 \mathrm{~min}$ and then $100 \mathrm{~V}$ for $80 \mathrm{~min}$ at $4{ }^{\circ} \mathrm{C}$ in TAE buffer containing $0.5 \mu \mathrm{g} / \mathrm{ml}$ of ethidium bromide.

Library. To detect pathogen DNA (here we targeted B. anthracis, F. tularensis, Ebola virus and SARS coronavirus), a small fragment of characteristic DNA sequences from the genome of each species was selected as the target DNA. Two separate sets of DNA probes, which were complementary to the two regions of the same target DNA, were synthesized. One blank control, where the two sets of probes were complementary to each other, was also chosen. Thus, a library (see Supplementary Table 5 online) of two sets of ssDNA probes (see Supplementary Table 2 online) were created. One set of probes (capture probes) was biotin-labeled and complementary to one part of its own target DNA. The other set of probes (report probes), which was complementary to the other part of the target DNA, was attached to the nanobarcodes, thus establishing the code library (see Supplementary Table 2 online).

Microbead functionalization with DNA probes. The conjugates between microbeads and DNA probes were prepared using a modified protocol suggested by the manufacturer (Bangs Laboratories). Briefly, $1.0 \mu \mathrm{g}$ of streptavidincoated polystyrene microbead suspension was washed with $100 \mu \mathrm{l}$ of TTL buffer (100 mM Tris-HCl, pH 8.0, 0.01\% Tween 20, $1 \mathrm{M} \mathrm{LiCl})$ and resuspended in $10 \mu \mathrm{l}$ of TTL. One picomole of biotin-modified capture probes was then mixed with the microbead suspension and incubated at $25{ }^{\circ} \mathrm{C}$ with gentle agitation for $30 \mathrm{~min}$. The excess and weakly bound probes were subsequently removed using sequential washes with $100 \mu \mathrm{l}$ of TTL buffer, TT buffer (250 mM Tris-HCl, pH 8.0, 0.01\% Tween 20), TTE buffer (250 mM Tris- $\mathrm{HCl}, \mathrm{pH} 8.0,0.01 \%$ Tween $20,20 \mathrm{mM} \mathrm{Na}_{2}$ (EDTA)) and TT buffer. The probe-functionalized microbeads were resuspended in prehybridization buffer (Church buffer: $0.5 \mathrm{M}$ sodium phosphate, $\mathrm{pH}$ 8.0, $1 \mathrm{mM}$ EDTA, 7\% (wt/vol) SDS and $1 \%(\mathrm{wt} / \mathrm{vol})$ bovine serum albumin) and incubated at $68{ }^{\circ} \mathrm{C}$ for $30 \mathrm{~min}$. After the prehybridization buffer was removed, the microbeads were resuspended in hybridization buffer $(1 \times$ SSC $(150 \mathrm{mM}$ sodium chloride, $15 \mathrm{mM}$ sodium citrate), $1 \%$ SDS). Other DNA probes were conjugated to microbeads similar to the method described above.

Sample preparation for fluorescence microscopy imaging. The sample for fluorescence microscopy analysis was prepared by thoroughly mixing bead/ probe conjugates along with a sample containing 'unknown' target(s) and nanobarcodes in $400 \mu \mathrm{l}$ of hybridization buffer to ensure uniform binding. The hybridization was performed with gentle agitation at $25^{\circ} \mathrm{C}$ in the dark for $2 \mathrm{~h}$. The sample was then washed with $400 \mu \mathrm{l}$ of hybridization buffer three times to remove excess and weakly bound nanobarcodes. The bead suspension was 
concentrated to a final concentration of 1,000 beads $/ \mathrm{ml}$. Around $2.5 \mu \mathrm{l}$ of concentrated bead suspension was added onto a glass slide. A cover slip was glued onto the glass slide using nail polish. After that, the sample was imaged with fluorescence microscopy at 1,000-fold magnification using green and farred filters, and the images were analyzed using MetaMorph software.

Dot blotting assay. Around $0.5 \mu \mathrm{l}$ of DNA solution (about $20 \mu \mathrm{M}$ ) including controls of a 15-mer oligonucleotide with irrelevant sequences and a $6.1 \mathrm{~kb}$ plasmid DNA was loaded onto a Zeta-probe membrane (Bio-Rad). After the membrane was air-dried, DNA molecules on the membrane were cross-linked o with a UV crosslinker (Stratagene). The membrane was then prehybridized with Church buffer at $68{ }^{\circ} \mathrm{C}$ for $2 \mathrm{~h}$. After prehybridization, the buffer was removed, and the membrane was submerged into a hybridization buffer $(1 \times$ SSC buffer containing 1\% SDS) containing nanobarcodes and incubated overnight at $25{ }^{\circ} \mathrm{C}$. After hybridization, the membrane was evaluated with fluorescence microscopy.

Sample preparation for flow cytometry. The microbeads used for flow cytometer analysis were purposely prepared nonuniformly in terms of the number of target DNA bound to each microbead (since only a small amount of target DNA was used, microbeads were far from saturation in terms of nanobarcode binding). Bead-probe conjugates, along with a sample containing 'unknown' DNA target(s) and nanobarcodes were added into $400 \mu \mathrm{l}$ of hybridization buffer individually without mixing to achieve a nonuniform nanobarcode binding. The resultant microbead suspension was incubated at $25^{\circ} \mathrm{C}$ in the dark for $2 \mathrm{~h}$. The sample was then analyzed using a flow cytometer (BD FACSCalibur) with green (FL1H) and far-red channels (FL4H).

Note: Supplementary information is available on the Nature Biotechnology website.

\section{ACKNOWLEDGMENTS}

We wish to acknowledge the Cornell Center for Advanced Technology and the Cornell Center for Vertebrate Genomics for financial support. This material is based upon work supported in part by the Science and Technology Center Program of the National Science Foundation under agreement no. ECS-

9876771. We thank Yung-Fu Chang for providing pathogen genomic DNA

(Mycobacterium avium subsp. paratuberculosis) and Carol Bayles for technical support on microscopy.

\section{COMPETING INTERESTS STATEMENT}

The authors declare competing financial interests (see the Nature Biotechnology website for details)

Received 22 February; accepted 5 May 2005

Published online at http://www.nature.com/naturebiotechnology/

1. Han, M., Gao, X.H., Su, J.Z. \& Nie, S. Quantum-dot-tagged microbeads for multiplexed optical coding of biomolecules. Nat. Biotechnol. 19, 631-635 (2001).

2. Fulton, R.J., McDade, R.L., Smith, P.L., Kienker, L.J. \& Kettman, J.R. Advanced multiplexed analysis with the FlowMetrix(TM) system. Clin. Chem. 43, 1749-1756 (1997).
3. Steemers, F.J., Ferguson, J.A. \& Walt, D.R. Screening unlabeled DNA targets with randomly ordered fiber-optic gene arrays. Nat. Biotechnol. 18, 91-94 (2000).

4. Braeckmans, K., De Smedt, S.C., Leblans, M., Pauwels, R. \& Demeester, J. Encoding microcarriers: Present and future technologies. Nat. Rev. Drug Discov. 1, 447-456 (2002).

5. Duggan, D., Bittner, M., Chen, Y., Meltzer, P. \& Trent, J. Expression profiling using cDNA microarrays. Nat. Genet. 21, 10-14 (1999).

6. DeRisi, J. et al. Use of a cDNA microarray to analyse gene expression patterns in human cancer. Nat. Genet. 14, 457-460 (1996).

7. Schena, M., Shalon, D., Davis, R. \& Brown, P. Quantitative monitoring of geneexpression patterns with a complementary-DNA microarray. Science 270, 467-470 (1995).

8. Cheung, V.G. et al. Making and reading microarrays. Nat. Genet. 21, 15-19 (1999).

9. Cunin, F. et al. Biomolecular screening with encoded porous-silicon photonic crystals. Nat. Mater. 1, 39-41 (2002).

10. Wang, J., Liu, G.D. \& Rivas, G. Encoded beads for electrochemical identification. Anal. Chem. 75, 4667-4671 (2003).

11. Chan, W.C. et al. Luminescent quantum dots for multiplexed biological detection and imaging. Curr. Opin. Biotechnol. 13, 40-46 (2002).

12. Nicewarner-Pena, S.R. et al. Submicrometer metallic barcodes. Science 294, 137 141 (2001).

13. Nam, J.M., Thaxton, C.S. \& Mirkin, C.A. Nanoparticle-based bio-bar codes for the ultrasensitive detection of proteins. Science 301, 1884-1886 (2003).

14. Li, Y. et al. Controlled assembly of dendrimer-like DNA. Nat. Mater. 3, 38-42 (2004).

15. Luo, D. The road from biology to materials. Mater. Today 6, 38-43 (2003).

16. Ried, T., Baldini, A., Rand, T.C. \& Ward, D.C. Simultaneous visualization of 7 different DNA probes by in situ hybridization using combinatorial fluorescence and digital imaging microscopy. Proc. Natl. Acad. Sci. USA 89, 1388-1392 (1992).

17. Taton, T., Mirkin, C. \& Letsinger, R. Scanometric DNA array detection with nanoparticle probes. Science 289, 1757-1760 (2000).

18. Sjostedt, A., Eriksson, U., Berglund, L. \& Tarnvik, A. Detection of Francisella tularensis in ulcers of patients with tularemia by PCR. J. Clin. Microbiol. 35, 1045-1048 (1997).

19. Sanchez, A. et al. Detection and molecular characterization of Ebola viruses causing disease in human and nonhuman primates. J. Infect. Dis. 179, S164-S169 (1999).

20. Poon, L.L. et al. Detection of SARS coronavirus in patients with severe acute respiratory syndrome by conventional and real-time quantitative reverse transcription-PCR assays. Clin. Chem. 50, 67-72 (2004).

21. Fuja, T., Hou, S. \& Bryant, P. A multiplex microsphere bead assay for comparative RNA expression analysis using flow cytometry. J. Biotechnol. 108, 193-205 (2004).

22. Vignali, D.A. Multiplexed particle-based flow cytometric assays. J. Immunol. Methods 243, 243-255 (2000)

23. Wang, J. et al. Adsorption and detection of DNA dendrimers at carbon electrodes. Electroanal. 10, 553-556 (1998).

24. Wang, J., Jiang, M., Nilsen, T. \& Getts, R. Dendritic nucleic acid probes for DNA biosensors. J. Am. Chem. Soc. 120, 8281-8282 (1998)

25. Nilsen, T., Grayzel, J. \& Prensky, W. Dendritic nucleic acid structures. J. Theor. Biol. 187, 273-284 (1997)

26. Capaldi, S., Getts, R.C. \& Jayasena, S.D. Signal amplification through nucleotide extension and excision on a dendritic DNA platform. Nucleic Acids Res. 28, e21 (2000).

27. Lowe, M. Spiro, A., Zhang, Y. \& Getts, R. Multiplexed, particle-based detection of DNA using flow cytometry with 3DNA dendrimers for signal amplification. Cytom Part A 60A, 135-144 (2004).

28. Shchepinov, M., Mir, K., Elder, J., Frank-Kamenetskii, M. \& Southern, E. Oligonucleotide dendrimers: stable nano-structures. Nucleic Acids Res. 27, 3035-3041 (1999).

29. Shchepinov, M., Udalova, I., Bridgman, A. \& Southern, E. Oligonucleotide dendrimers: synthesis and use as polylabelled DNA probes. Nucleic Acids Res. 25, 4447-4454 (1997). 